\title{
Molecular modeling of HSP90 and small molecules identification for drug development against paracoccidiodomycosis
}

\author{
Abadio, AKR ${ }^{1}$; Kioshima, ES²; Silva, PA 3 ; Nicola, $\mathrm{AM}^{3}$; Maigret, $\mathrm{B}^{4}$ and Felipe, $\mathrm{MSS}^{3}$. \\ ${ }^{1}$ Universidade do Estado de Mato Grosso, Department of Agronomy, Nova Mutum, Brazil. \\ 2Universidade Estadual de Maringá, Department of Medical Mycology, Maringá, Brazil. \\ ${ }^{3}$ Universidade de Brasilia, Department of Cellular Biology, Brasília, Brazil. \\ ${ }^{4}$ Université de Lorraine, Lorraine Research Laboratory in Computer Science and its Applications, Nancy, France.
}

The incidence and severity of systemic infections caused by fungi have increased around the world. Paracoccidioidomycosis, caused by Paracoccidioides spp., is an endemic systemic mycosis of Latin America, leading to serious and potentially fatal lungs, skin and lymph nodes. The heat shock protein of $90 \mathrm{kDa}$ (HSP90) act as molecular chaperone and contribute to fungal cellular processes including signal transduction, intracellular transport and protein degradation. The $h s p 90$ gene from Paracoccidioides lutzii is highly induced at the beginning of mycelium to yeast transition and remains at higher levels in the yeast form, making it a potential drug target. In order to develop new antifungal treatments against $P$. lutzii, we proposed to evaluate the target HSP90. We performed the homology modeling of the $\mathrm{N}$-terminus domain of $P$. lutzii HSP90 protein, the molecular dynamics simulations to obtain the model with stability and the virtual screening in chemical libraries for select the main small molecules that interact with HSP90 model. The 3D model of $P$. lutzii HSP90 was constructed by homology modeling based on known structures with high percentage of identity in amino acid sequences. The known template structures were searched in the Protein Data Bank. The amino acid residues sequences of HSP90 were compared with the primary sequences of the structures deposited in PDB using the BLAST program. The 3D model of HSP90 was constructed using the Insight II software package (Biosym/MSI, San Diego, Accelrys Inc. 2001). This model was further submitted to molecular dynamics simulations in order to gain a better relaxation and a more correct arrangement.
The NAMD program version 2.6 was employed in conjunction with the CHARMM22 force field. For virtual screening simulations, a bank of commercially available compounds from Life Chemicals database was docked with the model using GOLD program. The 3D model of $\mathrm{N}$-terminus domain of $P$. lutzii HSP90 protein was obtained and the molecular dynamics simulations revealed that the evolution of the system is very stable. The folding stability of the model during the simulations was checked by monitoring secondary structure conservation over simulation time. With the model stable, virtual screening was performed. In vitro activity antifungal assays were performed with the molecules selected after virtual screening using the broth microdilution methods developed by the Clinical and Laboratory Standards Institute. The small molecules that interact with the model were ranked and, among the best hits, 20 molecules were selected as putative inhibitors of HSP90. These molecules were synthesized for validation and in vitro activity antifungal assays for Paracoccidioides brasiliensis and $P$. lutzii. From 20 molecules tested, one of them (MOL90) showed antifungal activity in vitro against the fungi. In the absence of structures solved experimentally, the available homology modeling tools were extremely useful for the structural prediction of the HSP90 protein of $P$. lutzii. Moreover, the virtual screening allowed to identify a new antifungal compound against paracoccidioidomycosis. This work offered new perspectives on technological development and innovation of antifungal agents to these human pathogens.

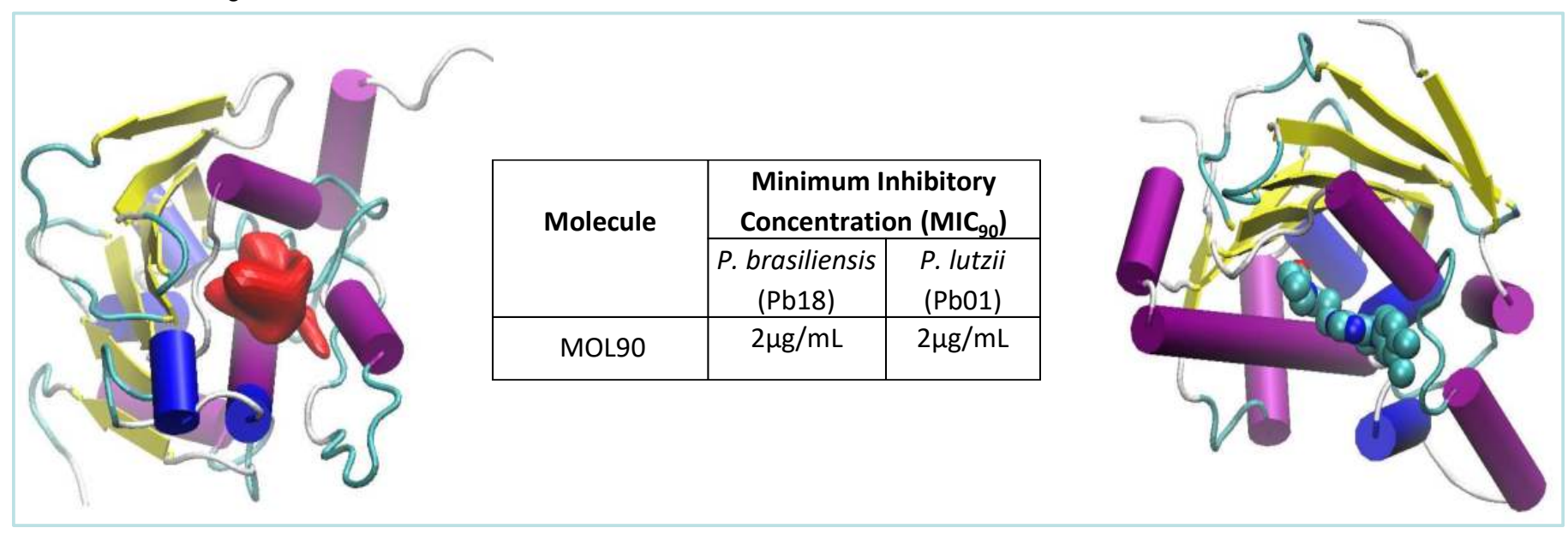

